\title{
Surface-enhanced Raman scattering and Plasmon effect for enzymatic bionanocomplexes characterization
}

\author{
Renata Wojnarowska-Nowak ${ }^{1}$, Jacek Polit $^{1}$, Daniel Broda ${ }^{2}$, Yaroslaw Bobitski ${ }^{3}$, Zbigniew Starowicz ${ }^{4}$, Mykhailo \\ Gonchar $^{2,5}$, and E. M. Sheregii ${ }^{1}$ \\ ${ }^{1}$ Centre for Microelectronics and Nanotechnology, University of Rzeszow, S. Pigonia 1, 35-959 Rzeszow, Poland \\ ${ }^{2}$ Department of Biotechnology, Institute of Applied Biotechnology and Basic Sciences, University of Rzeszow, \\ Werynia 502, 36-100 Kolbuszowa, Poland \\ ${ }^{3}$ Faculty of Mathematics and Natural Sciences, University of Rzeszow, S. Pigonia 1, 35-959 Rzeszow, Poland \\ ${ }^{4}$ Institute of Metallurgy and Materials Science Polish Academy of Sciences, Reymonta 25, 30-059 Krakow, Poland \\ ${ }^{5}$ Institute of Cell Biology, National Academy of Sciences of Ukraine, Drahomanov 14/16, Lviv 79005, Ukraine
}

\begin{abstract}
Cholesterol oxidase (ChOX) enzyme is one of the most important analytical enzyme, used for cholesterol assay in clinical diagnostics as well as food production, and the developing of innovative solutions for improving the selectivity and accuracy of the analysis including bio-nanotechnological approaches is still ongoing. The Surface Plazmon Resonance (SPR) and the surface enhanced Raman scattering (SERS) as specific for nanocurriers effects were observed what enable us to research the oscillation spectra of the ChOX enzyme. The vibrational lines are attributed to chemical functional groups existing in enzyme, for example, amino acids, amide groups as well as for cofactor. For the improving the SERS effect the gold nanoparticles - ChOX bionanocomplexes were analyzed in combination with goldcoating gratings as a promising plazmonic material.
\end{abstract}

\section{Introduction}

In the past few years surface enhanced Raman scattering (SERS) spectroscopy has been widely employed as a powerful and extremely sensitive technique [1]. Due to high sensitivity, selectivity and non-destructive data acquisition the method has been applied in chemistry and biochemistry [2]. Because of possibility to detect small amount of substances, or even single molecules, it can be employed successfully for analitical purposes [3]. SERS effect have been used in biological and medical applications, as well as environmental monitoring, through the development of optical biosensors $[4,5]$.

SERS signal can be promoted by means of surface plasmon resonance (SPR) and localized surface Plasmon resonances (LSPRs) [6,7]. Surface Plasmon's (SPs) are optical waves that originate from coupled collective oscillations of the electron plasma and associated electromagnetic field on metallic surface [1]. The SPR and LSPR phenomena can occur when chemical or biological elements interact with noble metal nanoparticles or nanostructures. The enhancing level is dependent on the composition, shape and size [8] of nanoparticles or nanostructures, as well as their geometry and roughens [9]. Occurrence of a strong SERS effect is also highly dependent on the interaction between investigated molecules and the surface of plasmonic nanostructures, which must be in close distance [7]. Therefore, it is important to develop the substrates which can get the maximum enchancing of the registered signal, while their production and using does not carry any major technical problems.

In presented work two kind of substrates were emplyed to obtained the plasmon effect: the gold nanoparticles (AuNPs) and polycarbonate gratings coated with gold, as a promising plasmonic materials $[5,10]$. The ChOX-AuNP bionanocomplex was situated on the grating which have improved optical properties and enhance the SERS effect additionally. The phenomenon of surface enhanced Raman scattering has been observed and has been used for the analysis of ChOX enzyme.

\section{Experiment}

\subsection{Sample preparation}

For the SERS study a bionanocomplex of cholesterol oxidase enzyme (ChOX, EC 1.1.3.6) and AuNP was performed [5]. The ChOX-AuNP bionanocomplexes were prepared by covalently binding of the gold nanoparticles and enzym via organic linker, as described in Ref. 5. AuNPs were obtained by modyfied Turkevich method with using chloroauric acid $\left(\mathrm{HAuCl}_{4}\right)$ and sodium citrate $\left(\mathrm{Na}_{3} \mathrm{C}_{6} \mathrm{H}_{5} \mathrm{O}_{7}\right)$ as a substrates of the reaction. On the surface of AuNP a mercaptohexa-

* Corresponding author: rwojnar@ur.edu.pl 
decanoic acid (MHDA) was immobilized as a linker for the ChOX enzyme binding.

In second step of presented study the prepared bionanocomplex was placed on the metal diffraction gratings as a plasmonic surfaces. They were made with using hologram replication technology [11] on the thin polycarbonate film. Grating depth is $300 \mathrm{~nm}$, with various grating period from $400 \mathrm{~nm}$ to $10000 \mathrm{~nm}$. The surface was covered by gold layer with $120 \mathrm{~nm}$ of layer thickness, by using vacuum deposition method.

\subsection{Methods of characterization}

The main methods for the ANPs and diffraction gratings characterization were two microscopic techniques: Atomic Force Microscopy (AFM, with using Innova Bruker microscope, in Tapping Mode), and Scanning Electron Microscopy (PhenomPro microscope).

The SERS effect was studied with using two spectrometers with different excitation sources: Smart Raman DXR Thermo Scientific spectrometer, with a $780 \mathrm{~nm}$ excitation wavelength (14 $\mathrm{mW}$ laser power), and InVia Micro Raman Renishaw spectrometer, with a 633 $\mathrm{nm}$ excitation (17 $\mathrm{mW}$ laser power).

\section{Results and discussion}

The AFM image of the AuNPs is shown in Fig. 1. The nanoparticles have spherical shape and size at about $45 \mathrm{~nm}$, however they are not completely homogenous. It is very difficult to obtain only one size of nanoparticles by the colloidal synthesis method. The size of AuNPs is dependent on the concentration of substrate and reducing agent in the synthesis mixture [5] as well as reaction conditions. After conjugation with enzyme the AuNPs are more aggregate and in various shapes but mainly spherical. It is seen in insert the assuming distribution of a near field for single spherical nanoparticle.

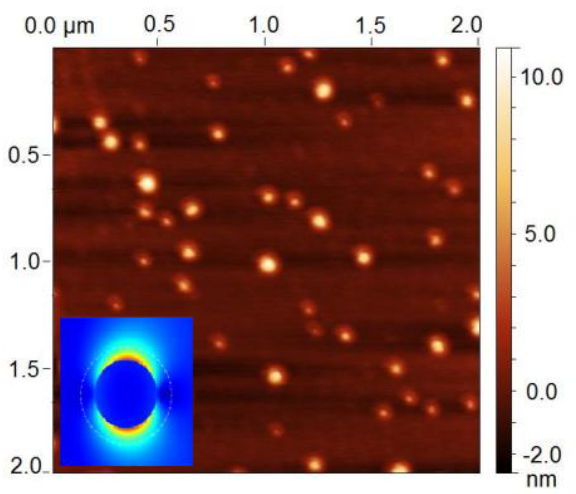

Fig 1. AFM imageing of AuNPs used for the synthesis of the bionanocomplex and distribution of a near field for single spherical nanoparticle (insert).

Topography of the prepared gold-coated polycarbonate grating is presented in Fig. 2. The grating period is determined to be $400 \mathrm{~nm}$. The groove depth after covering by gold layer is estimated to be about $120 \mathrm{~nm}$.
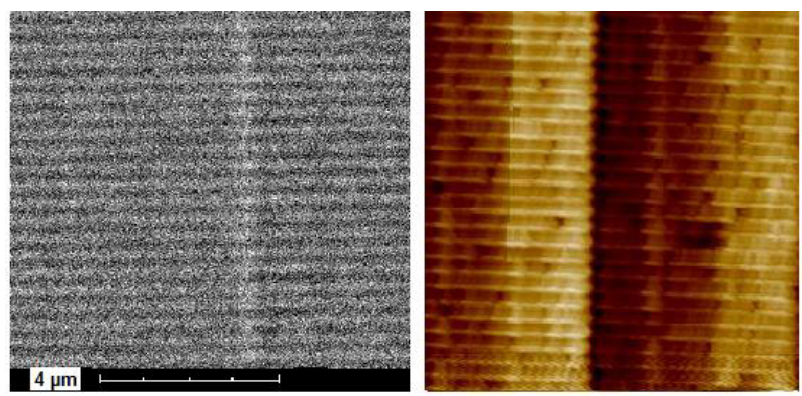

Fig. 2. SEM image (left) and AFM image (right) of goldcoated polycarbonate grating.

As was mentioned above ChOX enzyme is covalently immobilized with the AuNPs by MHDA linker and this connection is schematically shown in Fig. 3. The connection between the AuNPs and the MHDA linker is possible by presence of S-H thiol group and $\mathrm{Ag}-\mathrm{S}$ bond formation [8]. Carboxyl group of linker enables the protein binding by forming a peptide bond with the specified amino acids.

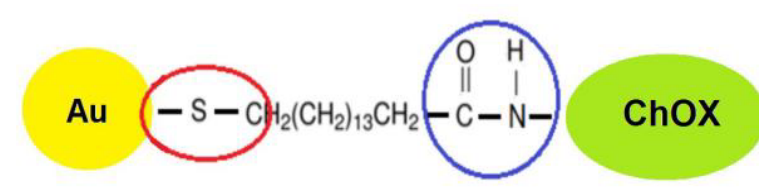

Fig. 3. Scheme of bionanocomplex consisting with AuNP, MHDA linker and cholesterol oxidase enzyme, with marked chemical bonds generated between them.

By this manner, the ChOX as an important analytical enzymes was investigated in conducted study. ChOX is used for the determination of the serum cholesterol level in clinical diagnostics [12,13]. It is an important biomarker of coronary heart disease, arteriosclerosis, and lipid metabolism dysfunction [14]. Furthermore, high concentration of cholesterol in food has a negative impact on our health, and because of this cholesterol level should be monitored in a food production [15]. Besides that $\mathrm{ChOX}$ is a promissing novel antibacterial therapeutic agent and herbicide [15]. Therefore, it is important also to develop convenient methods of controlling the presence of the enzyme.

The SERS effect has been observed for both of the prepared two bionanosystems. In Fig. 3 the Raman spectrum of the AuNP-ChOX bionanocomplex is shown (780 nm laser source). In Fig. 4 the Raman spectrum of bionanocomplex placed on the surface of gold-coated grating is presented (633 nm laser source).

Different degrees of the signal amplification (SERS) for prepared systems were achieved, which allows an optimization the architecture of prepared substrates. With using of gold-coating gratings with presented above parameters registered Raman signal was maximal.

Obtained Raman spectra can be used for the identification of functional groups existing in the ChOX enzyme [5]. Analogically to spectra obtained in Ref. 5, 
the most intense line observed at $1530 \mathrm{~cm}^{-1}$ in the Fig. 4. is assigned with the chemical bonds characteristic for proteins: N-H, C-N, and N-C-O vibrations, named as amide II $[16,17]$.

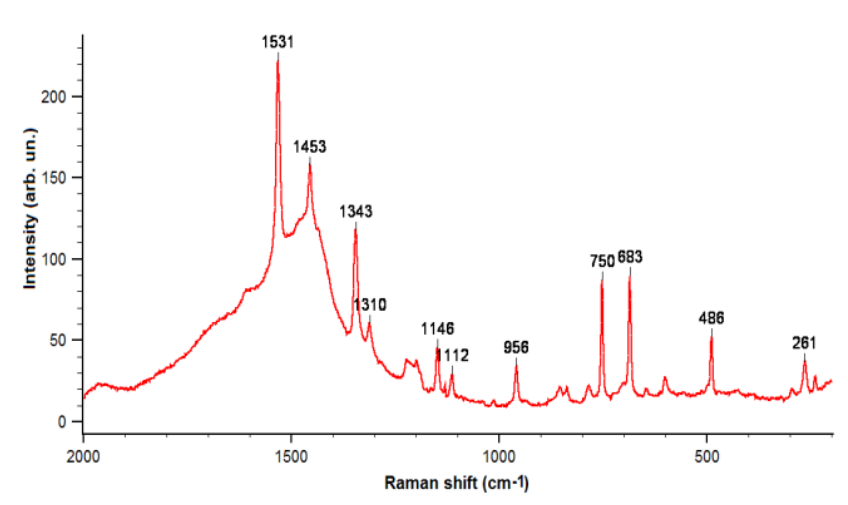

Fig. 4. SERS spectrum of AuNP-ChOX bionanocomplex, under $780 \mathrm{~nm}$ excitation wavelength.

Other bands characteristic for proteins are also observed. Amide I and III lines are recorded at $1610 \mathrm{~cm}^{-1}$ [16] and at around $1220 \mathrm{~cm}^{-1}$ [18], however they are significantly weaker. Other lines at $1146 \mathrm{~cm}^{-1}, 800-$ $900 \mathrm{~cm}^{-1}$ and $750 \mathrm{~cm}^{-1}$ can be attributed to the specific amino acids [19,20,21]. Some of the identified lines are not specific as a line at $1453 \mathrm{~cm}^{-1}$ associated witch $\mathrm{CH}$ vibration existing in linker and in protein structure as well [20]. Besides typical functional groups of proteins the vibration lines attributed to the Flavin adenine dinucleotide (FAD) cofactor have been observed (such as the adenine ring at 1343 and $1310 \mathrm{~cm}^{-1}$ [20], P-O around $1200 \mathrm{~cm}^{-1}$ and $\mathrm{C}=\mathrm{C}$ binding at $1112 \mathrm{~cm}^{-1}$ ) [18].

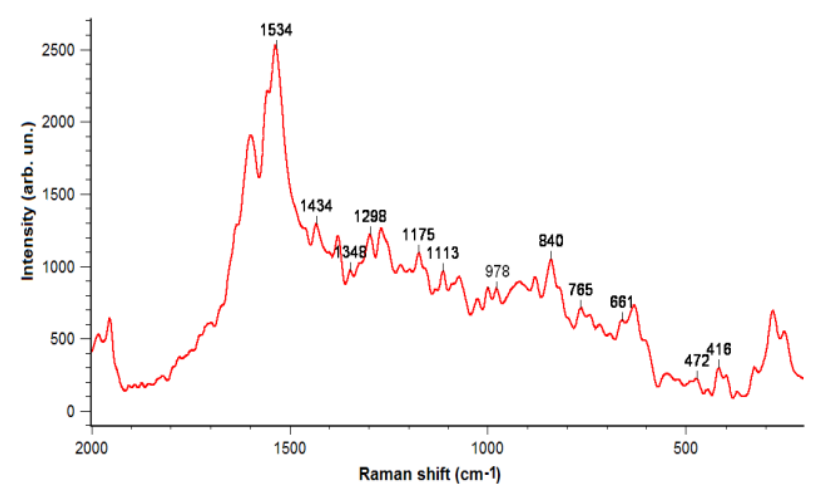

Fig. 5. SERS spectrum of AuNP-ChOX bionanocomplex placed on the gold grating, under $633 \mathrm{~nm}$ excitation wavelength.

The same characteristic lines can be observed in Fig. 5. However, the spectra obtained with investigated substrates are slightly modified. Some shifts of the bands, weakening of lines and strengthen others are observed. It can be cased by differen hotspot places in modyfied plasmonic system in combination with a high complication of protein molecule. The most intense line at $1531 \mathrm{~cm}^{-1}$, is slightly shifted to $1534 \mathrm{~cm}^{-1}$. Other lines attributed to amide I, amide II, some amino acids and
FAD cofactor are recorded also, and presented in Table 1.

Table 2. Identification of the lines observed in the Raman spectrum presented in Fig. 5.

\begin{tabular}{|c|c|c|c|}
\hline & $\begin{array}{c}\text { Position of } \\
\text { line }\left(\mathrm{cm}^{-1}\right)\end{array}$ & Identification & Reference \\
\hline 1 & 1602 & C=O band & 16,17 \\
\hline 2 & 1534 & N-H, C-N, N-C-O bands & 16,17 \\
\hline 3 & 1434 & C-H str. vibration & 17,20 \\
\hline 4 & 1349 & Adenine, ring I of FAD & 20 \\
\hline 5 & 1298 & C-N and N-H band & 16,17 \\
\hline 6 & 1264 & ring II of FAD molecules & 22 \\
\hline 7 & 1220 & C-N str., N-H & 18 \\
\hline 8 & 1113 & C=C of FAD & 18 \\
\hline 9 & 926 & C-C band & 17 \\
\hline 10 & $800-900$ & $\begin{array}{c}\text { arginine amino acid, } \\
\text { C-H out of plane }\end{array}$ & $19,20,21$ \\
\hline 11 & 765 & tryptophan & 21 \\
\hline
\end{tabular}

The resulting SERS effect suggests that bionanocomplexes AuNP-enzyme can be used for analytical purposes, and an optimisation of additional substrates to enchance the signal should be conducted.

\section{Conclusion}

The phenomenon of SERS effect was obtained using additional substrate of the gold covered diffraction grating. Prepared plasmonic surfaces could be usable SERS-platform for study of enzymes. Obtained SERS spectra can be used for the identification of functional groups existing in the examined enzyme or other biological molecules. Different degrees of the signal amplification (SERS) for prepared systems were achieved, which allows an optimization the architecture of prepared substrates. The gold-coated diffraction gratings in combination with a gold nanoparticlesenzyme bionanocomplexes can be perspective analytical instrument.

\section{References}

1. K.A. Willets, R.P. Van Duyne, Annu Rev Phys Chem. 58, 267 (2007)

2. S.Nie, S.R. Emory, Science 21, 275 (5303), 1102 (1997)

3. D.A. Stuart, J.M. Yuen, N. Shah, O. Lyandres, C.R. Yonzon, M.R. Glucksberg, J.T. Walsh, R.P. Van Duyne, Anal. Chem. 78 (20), 7211 (2006)

4. N.A. Mungroo, S. Neethirajan, Biosensors 21, 4, 472 (2014)

5. R. Wojnarowska, J. Polit, D. Broda, M. Gonchar, E. M. Sheregii, Appl. Phys. Let. 106, 103701 (2015)

6. H. Chen, X. Kou, Z. Yang, W. Ni, J. Wang, Langmuir 20, 24(10), 5233 (2008)

7. J. Ye, F. Wen, H. Sobhani, J.B. Lassiter, P. Van Dorpe, P. Nordlander, N.J. Halas, Nano Lett. 12, 3, $1660(2012)$ 
8. R. Wojnarowska, J. Polit, D. Broda, M. Gonchar, E. M. Sheregii, AMM 60, 3, 2289 (2015)

9. M.C. Estevez, M.A. Otte, B. Sepulveda, L.M. Lechuga, Anal. Chim. Acta. 806, 55 (2014)

10. B.K. Singh, A.C. Hillier, Anal. Chem. 80 (10), 3803 (2008)

11. M.T. Gale, Journal of imaging science and technology 41, 3, 211 (1997)

12. U. Saxena, M. Chakraborty, P. Goswami, Biosens. Bioelectron. 26, 3037 (2011)

13. L. Pollegioni, L. Piubelli, G. Molla, FEBS Journal, 23, 1742 (2009)

14. P. Srisawasdi, P. Jearanaikoon, N. Wetprasit, B. Sriwanthana, M.H. Kroll, P.H. Lolekha, Clin Chim Acta. 372, 103 (2006)

15. A. Vrielink, Subcell. Biochem. 51, 137 (2010)

16. J. Gelder, K. De Gussem, P. Vandenabeele, L. Moens, J. Raman Spectrosc. 38, 1133 (2007)

17. Rygula, A., Majzner, K., Marzec, K. M., Kaczor, A., Pilarczyk, M., Baranska, M., J. Raman Spectrosc. 44, 1061 (2013)

18. R. F. Brandao, R. L. Quirino, V. M. Mello, A. P. Tavares, A. C. Peres, F. Guinhos, J. C. Rubim, P. A. Z. Suarez, J. Braz. Chem. Soc. 20, 5, 954 (2009)

19. N. A. Atamas, A. M. Yaremko, T. Seeger, A. Leipertz, A. Bienko, Z. Latajka, H. Ratajczak, A. J. Barnes, J. Mol. Struct. 708, 189 (2004)

20. R. Tuma, J. Raman Spectrosc. 36, 307 (2005)

21. A. Brambilla, A. Philippidis, A. Nevin, D. Comelli, G. Valentini,D.Anglos, J. Mol. Struct. 1044, 121 (2013)

22. Zheng, Y., Care, P. R., Palfey, B. A., J. Raman Spectrosc. 35, 521 (2004) 